\title{
INFLUENCE DE L'INERTIE DE L'EAU SUR LA STABILITÉ D'UN GROUPE HYDROÉLECTRIQUE
}

\author{
P. ALMERAS \\ Ancien élève de l'École Polytechnique \\ Ingénieur aux Ateliers Neyret-Beylier \& Piccard-Pictet
}

\section{CAS DE PLUSIEURS GROUPES COUPLÉS EN PARALLÈLE}

Jusqu'ici nous avons traité de la stabilité d'un groupe alimentant seul un réseau séparé. Nous allons voir comment sont modifiées les conditions de stabilité, lorsque plusieurs groupes débitent en parallèle sur un même réseau $\left({ }^{2}\right)$. Le problème est évidemment complexe, aussi serons-nous obligés de faire quelques hypothèses simplificatrices, quitte à regarder dans quel sens il faudra modifier les résultats, compte tenu de la réalité. Précisons qu'il ne s'agit pas, ici, de la stabilité de la répartition de la charge entre les groupes, laquelle est obtenue par leurs statismes permanents, mais de l'étude des oscillations "qui permettent le passage d'un régime à un autre. Nous supposerons les statismes permanents nuls. Leur influence sur l'amortissement des oscillations est négligeable devant les autres influences (degré d'asservissement provisoire ou accéléromètre) .

HYPOTHESES. - I) Nous supposerons essentiellement que les lignes d'interconnexion et les installations électriques ont des caractéristiques telles que les liaisons entre centrales et récepteurs puissent être considérées comme rigides. Notons que nous avons implicitement fait cette hypothèse dans la marche en réseau séparé. Cette hypothèse était alors toujours justifiée par suite du peu d'étendue du réseau. Nous aurons une image mécanique de la chose en supposant tous les groupes attaquant un arbre rigide directement ou par l'intermédiaire d'engrenages, le tout actionnant un récepteur. Dans la réalité, les caractéristiques mécaniques des arbres de transmission et des engrenages (en particulier leur élasticité) jouent un rôle analogue aux caractéristiques électriques des lignes et des installations. Nous reviendrons sur la validité de cette hypothèse que nous considérons seulement comme une étape vers la résolution du problème dans le cas général.

II) Nous supposerons que pour chaque groupe on peut faire les hypothèses faites à l'occasion de la marche en réseau séparé (notamment indépendance des puissances motrices et résistantes à l'égard de la vitesse, et coup de bélier en masse).

III) Nous nous bornerons au cas de régulateur accélérotachymétrique ou à asservissement permanent, l'étude de l'asservissement temporaire ayant montré dans quelle mesure ce dernier gardait l'efficacité de l'asservissement permanent $\left({ }^{1}\right)$.

(I) Voir les numéros te et 2 (Janvicr et Mars 1946).

(2) On sait que la stabilité en réseau séparé d'une installation munie d'une cheninée d'équilibre, nécessite la réalisation d'une condition dite condition de Thoma. Or cette condition disparait dès que l'installation en guestion est couplée en parallèle sur un réseau de puissance au moins double de la sienne (voir article de M. Gaden, Schieiserische Banseitung des 30 juin et 6 juillet 1927). II aurait pu se faire que, comme la condition de Thoma, les conditions de staljilité du réglage yue nous ayons bouvées disparaissenl apres couplage sur un grand réseau. Nous vertnns futil n'en cat rieu.

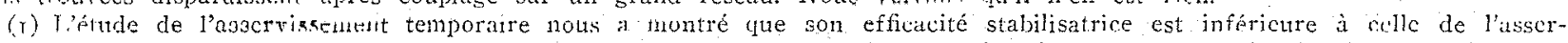

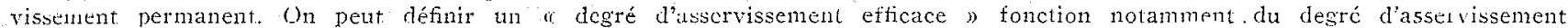
temporaire $\sigma$ de la rigidité du Dash pot ct de la rapidité de réponse $K_{n}$. Cc degré d'asservissturent efficace cst le legré d'asservisscment permancnt qui conférerait au système la même stabilité que le degré d'asservissement tomporaire o il est évidcmment infírienr à ee demier. Ce qui suit peut done s'appliquer à des régulitenrs a asservissement lunporaile, etant cntendu que les defres d'asservissencnt o domt il s'agit sont les degrés d'asservissement efficace. M. Gaden montre que, pour autant que la rapidité de réponse tachymétrique $K_{0}$ est très grande (et c'est ce qui est en fait rèalisé), le système d’ascervissement 
Dans ces conditions, les paramètres variables sont les suivants :

les $n$ ouvertures de vannage des $n$ groupes couplés,

les $n$ suppressions relatives dues au coup de bélier,

les $n$ vitesses de rotation des groupes.

Mais les $n$ vitesses de rotation, par suite de l'hypothèse du couplage rigide sur le réseau, sont proportionnelles à l'une d'elles choisie arbitrairement. Finalement, nous aurons, en général, un système à $2 n+1$ degré de liberté.

NOTATIONS. - Les groupes sont numérotés de 1 à $n$. Les indices $i$ se rapportent au groupe $i$ :

$\begin{aligned} \omega_{0 i} & \text { Vitesse de régime } \\ \omega_{i} & \text { Vitesse à l'instant } \\ \mathrm{I}_{i} & \text { Moment d'inertie } \\ \mathrm{P}_{0 i} & \text { Puissance de régime } \\ \mathrm{P}_{i} & \begin{array}{l}\text { Puissance au temps } t \text { sous chute nor- } \\ \text { male }\end{array} \\ \mathrm{P}_{p i} & \begin{array}{l}\text { Puissance de pleine ouverture sous } \\ \text { chute normale }\end{array} \\ \mathrm{P}_{p}=\Sigma \mathrm{P}_{p i} & \text { Puissance totale du réseau } \\ \mathrm{K}_{1 i i} & \text { Rapidité de réponse tachymétrique } \\ \mathrm{K}_{1 i} & \text { Rapidité de réponse accélérométrique } \\ \sigma_{i} & \text { Degré d'asservissement } \\ \zeta_{i} & \text { Suppression en temps } t \\ y_{0 i} & \text { Ouverture de régime }\end{aligned}$

$y_{i} \quad$ Ouverture au temps $t$

$\Theta_{i} \quad$ Temps caractéristique de la conduite à pleine ouverture

$\Theta_{0 i} \quad$ Temps caractéristique à l'ouverture $y_{0}$ $\Theta_{0 i}=y_{0} \Theta_{i}$

$\because \quad$ Temps de lancer du groupe

- Temps de lancer moyen des groupes $\tau=\frac{\sum_{i}^{\prime \prime} \mathrm{P}_{p i} \tau_{i}}{\mathrm{P}_{p}}$

$\hat{\xi}_{i}=y_{i}-y_{0 i} \quad$ Ecart relatif de vannage au temps $t$

$\xi=\frac{\left(\omega_{i}-\omega_{0 i}\right.}{\omega_{0 i}}$ Ecart relatif de vitesse au temps $t$ (le même pour tous les groupes par suite de l'hypothèse de la rigidité du couplage).

MISE EN ÉQUATION. — La loi de réponse s'écrit pour chaque groupe :

ou

$$
\frac{d \hat{s}_{i}}{d t}=-\mathrm{K}_{0 i}\left(\xi+\sigma_{i} \varepsilon_{i}\right)
$$

régulateur tachymétrique

$$
\frac{d s_{i}}{d t}=-\mathrm{K}_{0 i} \fallingdotseq-\mathrm{K}_{1 i} \frac{d \zeta}{d t}
$$

$$
\text { Le coup de bélier donne: } \quad \zeta_{i}=-\Theta_{i}\left(\frac{d \xi_{i}}{d t}+\frac{1}{2} y_{0} \frac{d \xi_{i}}{d t}\right)
$$
régulateur accélérotachymétrique

En appliquant l'équation fondamentale du mouvement :

$$
\Sigma I_{i} \frac{\left.\omega_{i} d \omega\right)_{i}}{i d t}=\Sigma P \text { mutrices }-\Sigma P \text { résistantes }
$$

il vient facilement après linéarisation :

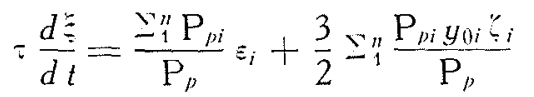

alors que pour chaque groupe $i$ fonctionnant en réseau séparé, l'équation de mouvement serait :

$$
\tau_{i} \frac{d \xi_{i}}{d t}=\xi_{i}+\frac{3}{2} y_{0 i} \xi_{i}
$$

dans lc fonctionnement en parallàlc, le système (1) qui régit le mouvemont comprend $2 n+1$ ćquation, soit :

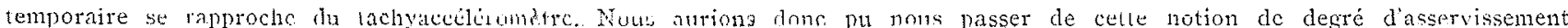
efficace. Nous avons prefére la garder car le deyré d'asscrvissement effrace peut servit de mestre a lat linesse lu léglage de la fréquence du résean. A mue variation de puissance $\varepsilon_{0}$ correspond en effet une variation relative de fríluence $\xi_{0}$ proportionnelle à $\sigma \varepsilon_{0}$ 


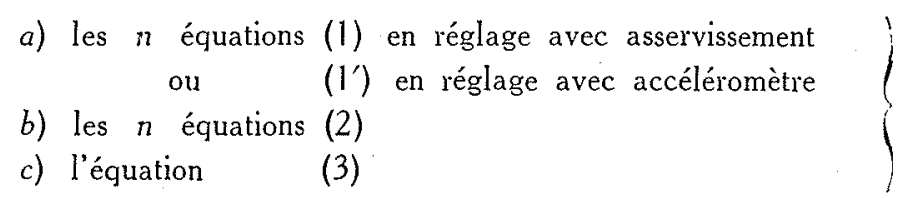

Le mouvement du groupe $i$ fonctionnant en réseau séparé serait régi par le système (I $i$ ) qui comprend trois équations, soit :
a) l'équation (1) en réglage avec asservissement ou (1') en réglage avec accéléromètre
b) l'équation (2)
c) l'équation $(3 i)$

L'équation caractéristique du système (I i) peut s'écrire (voir numéro de Novembre 1945) :

$$
\begin{gathered}
\Phi_{i}(r) \equiv \tau_{i} r+\frac{K_{0 i}\left(1-\Theta_{0 i} r\right)}{\left(r+K_{0 i} \sigma_{i}\right)\left(1+\frac{\Theta_{0 i} r}{2}\right)}=0 \\
\Phi_{i}^{\prime}(r) \equiv i^{i} r^{2}+\left(K_{0 i}+K_{1 i} r\right) \frac{1-\Theta_{0 i} \mathrm{r}}{1+\frac{\Theta_{0 i} r}{2}}=0
\end{gathered}
$$

réglage avec asservissement

réglage avec accéléromètre

On trouve que l'équation caractéristique du système (I $i)$ s'écrit tous calculs faits :

ou

$$
\begin{aligned}
& \Phi(r) \equiv \tau+\frac{1}{\mathrm{P}_{\rho}} \Sigma_{1}^{n} \frac{\mathrm{P}_{p i} \mathrm{~K}_{0 i}\left(1-\Theta_{0 i} r\right)}{\left(r+\mathrm{K}_{0 i} \sigma_{i}\right)\left(1+\frac{\Theta_{0 i} r}{2}\right)}=0 \\
& \Theta^{\prime}(r) \equiv \tau r^{2}+\frac{1}{\mathrm{P}_{\rho}} \Sigma_{1}^{n} \mathrm{P}_{p i}\left(\mathrm{~K}_{0 i}+\mathrm{K}_{1 i} r\right) \frac{1-\Theta_{0 i} r}{1+\frac{\Theta_{0 i} r}{2}}=0
\end{aligned}
$$

réglage avec asservissement

réglage avec accéléromètre

c'est-à-dire que de même qu'on passe de l'équation $(3 i)$ à l'équation (3) par multiplication par $P_{p i}$ et sommation, on passe de $\Phi_{i}(r)$ à $\Phi(r)$ par multiplication par $P_{p i}$ et sommation : $\Phi(r) \equiv \frac{\sum \mathrm{P}_{p i} \Phi_{i}(r)}{\mathrm{P}_{p}}$

CONDITIONS DE STABILITE. - Les équations caractéristiques (5) et $\left(5^{\prime}\right)$ sont $(2 n+1)^{\circ}$ degré en $r$. L'application des conditions d'Hurwitz ne leur est guère possible. Aussi devons-nous nous borner à traiter des cas particuliers en nombre suffisant et pour lesquels leur application est possible; ceci nous donnera suffisamment d'éléments pour répondre à la question posée. Pour simplifier l'exposé, dans chaque cas particulier, nous nous bornerons à l'étude du cas où les régulateurs des $n$ groupes sont stabilisés par asservissement.

Le cas où tous les groupes ont des régulateurs accélérot achymétriques est en général plus simple à traiter et conduit aux mêmes conclusions générales.

${ }^{\mathrm{Hr}}$ Cas. - Tous les temps caractéristiques des conduites $\Theta_{0 i}$ sont égaux à $\Theta_{0}$, c'est-à-dire qque tous les groupes sont également soumis à l'influence de l'inertie de l'eau.

D'autre part, comme nous avons vu que dans un régulateur à asservissement il y a intérêt à avoir $K_{0}$ grand, nous supposerons $K_{0}$ infini. De ce fait, dans l'équation (5) le facteur : $\frac{r+K_{0 i} \sigma_{i}}{K_{0}}$ est remplacé par : $\frac{1}{\sigma_{1}}$

L'équation (5) s'écrit :

$$
\tau r+\frac{1-\Theta_{0} r}{1+\frac{\Theta_{0} r}{2}} \frac{1}{\mathrm{P}_{p}} \Sigma \frac{\mathrm{P}_{p i}}{\sigma_{i}}=0
$$


L'équation (4) s'écrit de son côté : $\quad \tau_{i} r+\frac{1-\Theta_{0} r}{1+\frac{\Theta_{0 r}}{2}} \frac{1}{\tau_{i}}=0$

La comparaison de ces deux équations montre que l'ensemble des groupes couplés se comporte comme un seul groupe qui aurait comme temps de lancer la moyenne des iemps de lancer pondérés par la puissance des groupes:

$$
\tau=\frac{1}{\mathrm{P}_{p}} \sum_{1}^{n} \mathrm{P}_{p i} \tau_{i}
$$

et pour degré d'asservissement, la moyenne harmonique des degrés d'asservissement pondérés par les puissances des groupes.

$$
\frac{1}{\sigma}=\frac{1}{P_{p}} \sum_{1}^{n} \frac{P_{p i}}{\sigma_{i}}
$$

La condition de stabilité de chaque groupe en réseau séparé s'écrit :

$$
\tau_{i} \sigma_{i}>\Theta_{0} \quad \text { ou } \quad P_{p i} \tau_{i}>\Theta_{0} \frac{P_{p i}}{\sigma_{i}}
$$

La condition de stabilité de l'ensem'jle couplé :

$$
\tau>\Theta_{0} \quad \text { ou } \quad \tau>\theta_{0} \frac{1}{\sigma}
$$

On passe de l'une à l'autre des conditions de stabilité par sommation.

Donc, si chaque groupe est en réseau séparé, à la limite de la stabilité l'ensemble couplé sera aussi à la limite de stabilité. L'interconnexion ne crée pas, par elle-même, de stabilité. Elle permet simplement une répartition quelconque des inerties et des degrés d'asservissement des groupes.

Dans tout ce qui suit, quand nous parlerons de moyennes pondérées, il sera sous-entendu que les coefficients de pondération sont les puissances maxima des groupes.

Avec des régulateurs accélérotachymétriques on trouve que le groupe équivalent a le temps de lancer $\tau$ (moyenne pondérée des temps de lancer) et que ses rapidités de réponses tachymétriques et accélérométriques sont les moyennes pondérées des rapidités de réponse des groupes, ce qui conduit à la même conclusion que ci-dessus.

$2^{e}$ Cas. - Les temps caractéristiques des conduites sont quelconques, mais le premier groupe, par exemple, est seul à régler, les autres étant sur limiteur d'ouverture ou ayant un statisme infini (ce qui se traduit par $\mathrm{K}_{0 i}=0$ ou $\sigma_{i}=\infty$ pour un régulateur à asservissement et par $\mathrm{K}_{0 i}=\mathrm{K}_{1 i}=0$ pour un régulateur accélérotachymétrique).

Notons qưe cela ne veut pas dire forcément que les ouvertures de ces groupes sont constantes, mais seulement qu'elles sont fonction d'un autre paramètre que la vitesse (par exemple le limiteur d'ouverture peut être asservi à un niveau d'eau ou à une puissance de programme).

L'ćquation (5) s'écrit :

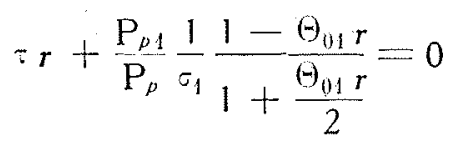

L'ćquation (4) s'ćcrit de son côtć, pour lc groupc 1 :

$$
\pi_{1} r+\frac{1}{\sigma_{1}} \frac{1-\Theta_{01} r}{1+\frac{\Theta_{01} r}{2}}=0
$$

(I) On voit apparaitre ici lc paramètre $\frac{P_{p i}}{c_{i}}$ que nous pourrions appeler puissance réglante temporaire du groupe $i$; cette notion est, au facteur fréquence près, analosue à la notion d'énergie réglante introduite par M. Darrieus. Toutefois, il s’agit ici de puissance réglante "temporaire" en ce scons que le degré dasservissement ou statisme qui lui est associé est un statisme " temporaire ». 
La condition de stabilité de l'ensemble s'écrit :

tandis que celle du groupe 1 en réseau séparé s'écrit :

$$
\tau \sigma_{1}>\frac{P_{p 1}}{P_{p}} \Theta_{01}
$$

$$
\tau_{1} \sigma_{1}>\Theta_{01}
$$

La comparaison de ces deux conditions montre que le simple fait d'avoir mis une certaine fraction $1-\frac{\mathrm{P}_{p 1}}{\mathrm{P}_{p}}$ de la puissance sur limiteur d'ouverture a, pour effet, de multiplier fictivement le temps de lancer moyen par $\frac{\mathrm{P}_{p}}{\mathrm{P}_{p 1}}>1$ donc de stabiliser le réseau. On peut aussi bien dire que la mise d'une partie des groupes sur limiteur d'ouverture a pour effet de multiplier fictivement par $\frac{\mathrm{P}_{p}}{\mathrm{P}_{p 1}}>1$ le degré d'asservissement des groupes régleurs.

On remarque que ce deuxième cas est en partie contenu dans le premier.

$\mathrm{Si}$, en effet, dans le premier on rend infinis les degrés d'asservissement des $n-1$ derniers groupes, on retombe sur le résultat précédent. Mais il est évident qu'alors, on n'a pas besoin de faire, comme nous avons dû le faire dans le premier cas, l'hypothèse de l'égalité des $\Theta_{0 i}$ : les $\Theta_{0 i}$ des groupes sur limiteur d'ouverture n'interviennent pas.

3e Cas. - Les temps caractéristiques des conduites sont quelconques.

L'équation (5) devient :

$$
\tau r+\frac{1}{P_{p}} \Sigma_{1}^{n} \frac{P_{p i}}{\sigma_{1}} \frac{1-\Theta_{0 i} r}{1+\frac{\Theta_{0 i} r}{2}}=0
$$

Son degré est $n+1$. Si nous ne voulons pas que les conditions d'Hurwitz deviennent inextricables, nous devons nous borner au cas de deux groupes: $P_{1} \tau_{1} \sigma_{1} \Theta_{1}$ et $P_{2} \tau_{2} \sigma_{2} \Theta_{2}$, la puissance totale étant $P_{1}+P_{2}$.

L'équation caractéristique (5) devient alors :

$$
\mathrm{Pr}+\frac{\mathrm{P}_{1}}{\sigma_{1}} \frac{1-\Theta_{1} r}{1+\frac{\Theta_{1} r}{2}}+\frac{\mathrm{P}_{2}}{\sigma_{2}} \frac{1-\frac{\Theta_{2} r}{1+\frac{\Theta_{2} r}{2}}}{1+0}=0
$$

de la forme :

$$
\begin{aligned}
& r^{3}+a r^{2}+b r+c=0 \\
& a=\frac{P-\frac{\Theta_{1}+\Theta_{2}}{2}-\frac{\Theta_{1} \Theta_{2}}{2}\left(\frac{P_{1}}{\sigma_{1}}+\frac{P_{2}}{\sigma_{2}}\right)}{P-\frac{\Theta_{1} \Theta_{2}}{4}} \\
& b=\frac{P=-\frac{P_{1}}{\sigma_{1}}\left(\Theta-\frac{\Theta_{2}}{2}\right)-\frac{P_{2}}{\sigma_{2}}\left(\Theta_{2}-\frac{\Theta_{1}}{2}\right)}{P=\frac{\Theta_{1} \Theta_{2}}{2}} \\
& c=\frac{\frac{P_{1}}{\sigma_{1}}+\frac{P_{2}}{\sigma_{2}}}{P_{\tau} \frac{\theta_{1} \theta_{2}}{4}}
\end{aligned}
$$

Les conditions d'Hurwitz sont:

$$
a>0 \quad c>0 \quad a b-c>0
$$


La condition $c>0$ est toujours réalisée. Les deux autres s'écrivent :

$$
\begin{gathered}
P_{-} \frac{\Theta_{1}+\Theta_{2}}{2}-\frac{\Theta_{1} \Theta_{2}}{1}\left(\frac{P_{1}}{\sigma_{1}}+\frac{P_{2}}{\sigma_{2}}\right)>0 \\
\text { et } \left.\left[P=\frac{\Theta_{1}+\Theta_{2}}{2}-\frac{\Theta_{1} \Theta_{2}}{2}\left(\frac{P_{1}}{\sigma_{1}}+\frac{P_{2}}{\sigma_{2}}\right)\right]\left[P-\frac{P_{1}}{\sigma_{1}}\left(\Theta_{1}-\frac{\Theta_{2}}{2}\right)-\frac{P_{2}}{\sigma_{2}} \Theta_{2}-\frac{\Theta_{1}}{2}\right)\right]>P-\left(\frac{P_{1}}{\sigma_{1}}+\frac{P_{2}}{\sigma_{2}}\right) \frac{\Theta_{1} \Theta_{2}}{4}
\end{gathered}
$$

Nous sommes donc conduits à des conditions assez complexes. Cependant, ce qui nous importe, c'est de savoir si, chacun des groupes étant à sa limite de stabilité en réseau séparé, ils y sont encore après couplage, ou si, au contraire, le couplage crée ou non de la stabilité.

Supposons les deux groupes séparément à la limite de stabilité; dès lors, vu les hypothèses, on a :

$$
\tau_{1} \sigma_{1}=\theta_{1} \quad \tau_{2} \sigma_{2}=\theta_{2}
$$

La première condition devient alors: $P_{1} \tau_{1}{ }^{2} \sigma_{1}+P_{2} \tau_{2}{ }^{2} \sigma_{2}>0$ automatiquement réalisée.

Reste la deuxième qui, tous calculs faits, et à un facteur positif près, s'écrit :

$$
\left(\frac{\Theta_{1}}{\Theta_{2}}-1\right)\left(\frac{\Theta_{1}}{\Theta_{2}}-\frac{\sigma_{2}}{\sigma_{1}}\right)>0
$$

Nous retrouvons ici le fait que si $\Theta_{1}=\mathrm{H}_{2}$ on se trouve encore à la limite de stabilité, après couplage. De même si, forfuitement, l'égalité $\Theta_{1} \sigma_{1}=\Theta_{2} \sigma_{2}$ est réalisée (degié d'asservissement $\sigma$ inversement proportionnel aux $\Theta$ ).

Supposons maintenant que $\Theta_{1}$ soit supérieur à $\Theta_{2}$ ou $\frac{\Theta_{1}}{\Theta_{2}}>1$

si : $\quad 1<\frac{\Theta_{1}}{\Theta_{2}}<\frac{\sigma_{2}}{\sigma_{1}}$ l'inégalité n'est pas satisfaite,

si : $\quad \frac{\sigma_{2}}{\sigma_{1}}<\frac{\Theta_{1}}{\Theta_{2}}$ l’inégalité est satisfaite,

donc si : $\quad 1<\frac{\Theta_{1}}{\Theta_{2}}<\frac{\sigma_{2}}{\sigma_{1}}$ les deux groupes deviennent instables après couplage,

si : $\quad 1<\underset{\Theta_{2}}{\Theta_{1}}<\frac{\sigma_{2}}{\sigma_{1}}$ les deux groupes deviennent stables après couplage.

Cependant, il ne faut pas accorder une grande importance à ces influences car le calcul montre que, vu les valeurs habituelles des différents paramètres, le gain ou la perte de $P d^{2}$ (1) qui peut en résulter ne dépasse guère 5 à $10 \%$.

D'ailleurs, lorsqu'un grand nombre de groupes sont couplés en parallèle, on peut penser que ces influences contraires se compensent, de sorte qu'au total il n'y a ni gain ni perte de stabilité.

CONCLUSIONS. - Ces quelques exemples nous suffisent pour affirmer que :

Lorsque plusieurs groupes sont couplés sur un réseau, l'ensemble se conduit, en quelque sorte, comme un seul groupe dont les caractéristiques (d'inerties, de régulateurs, de conduites) seraient des caractéristiques moyennes de celles des groupes couplés.

Si nous négligeons les faibles influences dont il est question au $3^{\circ}$ cas, nous pouvons énoncer les propositions suivantes :

$1^{\circ)}$ Lorsque plusieurs groupes sont stables en fonctionnement séparé, leur ensemble reste stable après couplage. couplage.

$\left.2^{\circ}\right)$ Lorsque plusieurs groupes sont instables en fonctionnement séparé, leur ensemble reste instable après

$\left.3^{\circ}\right)$ L'ensemble de plusieurs groupes couplés peut êire stable, même si certains groupes sont instables en réseau séparé, à condition que les autres aient unc margc dc stabilité suffisante.

Nous arrivons ainsi aux résultats auxquels M. CxADF,N arrive dans son ouvrage.

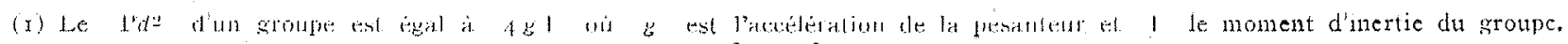

$$
\text { par la formule } \tau=\frac{\mathrm{P} d^{2} \times n^{2}}{270,000, \mathrm{~N}} \text { ou } \mathrm{P} d^{2} \text { est en kg.m², } n \text { en tours-minute, et } \mathrm{N}
$$


L'interconnexion ne crée donc pas, par elle-même, de la stabilité. $\mathrm{Si}$ on a pu constater expérimentalement que des groupes instables en réseau séparé peuvent être utilisés sans inconvénient après couplage sur un grand réseau, c'est que le réseau en question possédait une marge de stabilité suffisante.

D'autre part, le couplage sur un réseau stable d'un groupe instable, diminue la marge de stabilité de l'ensemble et on ne pourrait indéfniment coupler sur un réseau stable des groupes instables, sans aboutir, finalement, à rendre le réseau lui-même instable.

Les calculs précédents montrent que le couplage d'un groupe instable sur un réseau stable affecte d'autant plus la stabilité de ce dernier, que ce groupe est plus puissant. Pour nous en tenir aux régulateurs à asservissement nous avons vu, en effet, que le groupe équivalent à l'ensemble des groupes couplés avait un degré d'asservissement égal à la moyenne harmonique des degrés d'asservissement pondérés par. les puissances des groupes.

Ceci dit, à quoi est due la grande marge de stabilité du réseau d'interconnexion français, en particulier? La réponse n'est pas difficile : une grande partie de la puissance hydroélectrique française est au fil de l'eau et les groupes qui la fournissent ont leurs régulateurs le plus souvent sur limiteur d'ouverture. On a aussi placé sur limiteur d'ouverture les régulateurs de quantité d'installations anciennes, régulateurs qui, ayant pris du jeu, étaient devenus instables. D'autre part, un certain nombre de centrales à réservoir sont mises sur limiteur, de façon à réaliser un programme de vidange, et le réglage de la fréquence est principalement réalisé par les usines à réserve journalière. Or, tous ces groupes sur limiteur d'ouverture sont équivalents à des groupes de statisme infini (degré d'asservissement infini) et contribuent à augmenter considérablement le degré d'asservissement du groupe équivalent et, par conséquent, la stabilité du système. On pourrait dire aussi, et la conclusion serait la même, que les groupes sur limiteur d'ouverture augmentent fictivement le temps de lancer moyen du groupe équivalent. $\mathrm{Si}$ même tous les groupes se trouvaient sur limiteur d'ouverture, le réseau serait parfaitement stable, mais alors la fréquence ne serait plus du tout réglée.

On voit apparaître, là encore, l'antagonisme déjà remarqué à propos de la marche en réseau séparé, entre la stabilité de réglage et sa finesse. On peut dire qu'en gros, l'écart de fréquence $\xi_{0}$ qui suit un écart relatif $\varepsilon_{0}$ de la puissance demandée par le réseau, varie comme le degré d'asservissement moyen du réseau (en réglage tachymétrique).

Il faut considérer cependant, comme l'ont fait remarquer MM. Aillener et Chenais lors de la séance du 10 février 1944 de la Commission d'étude de la limite de puissance des machines hydrauliques, qu'on peut se permettre de ne pas faire régler ou de faire peu régler une fraction d'autant plus grande de la puissance du réseau, que la puissance totale de ce réseau est elle-même plus grande. Cela tient au fait que la puissance unitaire des installations utilisatrices rapportée à la puissance totale, décroît quand la puissance du réseau crôit. Dès lors, les variations brusques relatives de la puissance demandée (arrêt ou mise en marche d'un groupe utilisateur) sont plus faibles sur un grand que sur un petit réseau. Par suite sur un grand réseau, on pourra maintenir la fréquence avec la même précision, tout en ayant un degré d'asservissement moyen plus grand - en réglage tachymétrique - que sur un petit réseau. Pour essayer de préciser cette notion, nous pouvons faire un raisonnement faisant appel au calcul des probabilités et qui nous a été suggéré par M. CRAYA.

Considérons un réseau quelconque. La puissance demandée par les utilisateurs comporte des variations systématiques (pointes, etc.) et des variations aléatoires (dues à la fantaisie des usagers). Les premières sont prévisibles et sont compensées par une variation correspondante de la puissance fournie, prévue au programme. Nous ne nous intéresserons donc qu'à la deuxième, celle qui produit la «dentelle» des variations de fréquence du réseau.

A un instant donné, la valeur de la puissance à fournir $p_{0}$ prévue au programme est - si ce programme est bien fait - la valeur la plus probable de la puissance demandée en cet instant. Pour faciliter l'exposé, nous supposerons que sont seules possibles des demandes de puisance $p_{0} p_{0} \pm \lambda \ldots p_{0} \pm i \lambda \ldots$ discontinues.

Portons en abscisses ces valeurs possibles de la puissance demandée, et en ordonnée les probabilités correspondantes (fig. 13). Ceci nous donne la courbe ${ }^{(1)}$ discontinue $\pi$ des probabilités des puissances demandées, dont nous ne savons rien à priori, si ce n'est qu'elle comporte un maximum $\pi_{0}$ pour la puissance $p_{t)}$, que $\pi$ est petit pour $i$ grand et que $\Sigma \pi_{i}=1$.

La probabilité $\Pi$ pour qu'à un instant donné il y ait une variation brusque de puissance $\mathrm{K} \lambda$ d'une valeur quelconque $p_{i}$ à une autre $p_{i}+\mathrm{K} \lambda$ est la somme - étendue à toutẹs les $i$ - des produits $\pi_{i} \mid \mathrm{K}$.

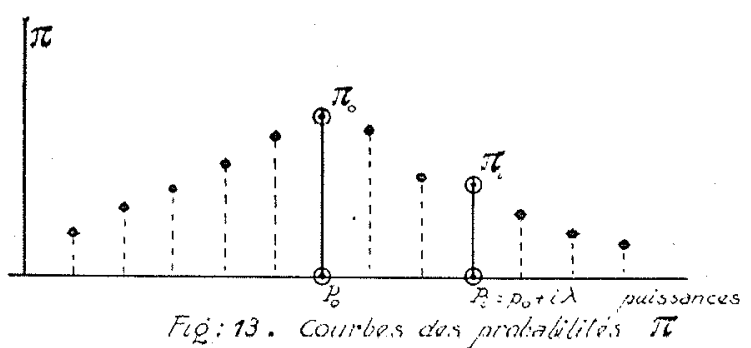

(I) C peut être construile grâce à une enquête statistique répartie sur un espace de temps suffisamment long. 
On pourra tracer (fig. 14) en portant en abscisse $\mathrm{K} \lambda$ et en ordonnée les $\Pi$ la courbe $I_{\mathrm{K}}$ de ces probabilités qui est, en somme, la transformée de la courbe de la fig. 13 par l'opération précédente.

On voit, facilement, que cette probabilité présente un maximum pour $K=0$ et qu'elle est nulle pour $K$ grand.

\section{Considérons l'expression}

$$
a^{2}=\Sigma_{K} I_{K} K^{2} \lambda^{2}
$$

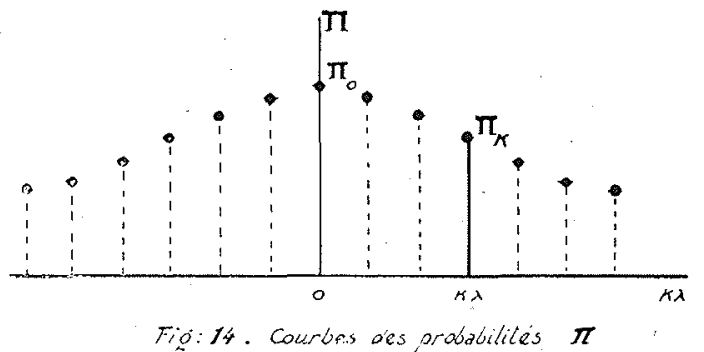

de l'écart quadratique moyen relatif à cette courbe. Il définit,

en quelque sorte, le caractère plus ou moins ramassé (autour de son maximum) de la courbe des probabilités II des diverses variations brusques possibles, de la même manière que le rayon de giration d'un solide caractérise le groupement plus ou moins ramassé de ce solide autour du centre de gravité (Paul Lévy). La valeur de $\alpha$ fournit l'ordre de grandeur des variations de puissance $\Delta p$ les plus fréquentes. La variation de fréquence qui suit une variation $\Delta p$ de la puissance demandée est, en gros, proportionnelle à cette variation rapportée à la puissance fournie, soit $\Delta p / \mathrm{p}_{0}$. Donc, on peut dire que la finesse du réglage de la fréquence d'un réseau est inversement proportionnelle à $\alpha / p_{0}$.

Considérons maintenant $n$ réseaux identiques - tant dans leurs puissances que dans les fantaisies de leur clientèle -. Pour chacun d'eux on peut défnir les courbes de probabilités $\pi$ et $\Pi$ qui seront identiques aux précédentes. Couplons ces $n$ réseaux en un réseau total de puissance $P_{0}=n p_{0}$. A un instant donné, la variation brusque de la puissance demandée sur le réseau total est égale à la somme des $n$ variations brusques des puissances demandées par les réseaux partiels, dont les probabilités sont données par les $n$ courbes. ПI identiques. Soit la courbe $\Pi$ correspondant au réseau total et soit A l'écart quadratique moyen correspondant, on sait (Loi des erreurs résultantes) que l'on a :

ou

$$
\begin{gathered}
\mathrm{A}^{2}=n \alpha^{2} \quad \text { ou } \quad \mathrm{A}=\alpha \sqrt{n} \\
\frac{\mathrm{P}_{0}}{\mathrm{~A}}=\frac{a}{\mathrm{P}_{0}} \sqrt{n}=\frac{a}{\mathrm{P}_{0}} \frac{1}{\sqrt{n}}
\end{gathered}
$$

Il s'ensuit que la finesse de réglage d'un réseau est proportionnelle à la racine carrée de sa puissance : lorsqu'on couple entre eux deux réseaux de même puissance et ayant tous deux même marge de stabilité (même amortissement), le réseau résultant a la même marge de stabilité, mais la finesse de réglage (caractérisée par $\frac{A}{P_{0}}$ ) est $\quad \sqrt{2}$ fois plus grande.

Ceci explique, ce qu'on a remarqué depuis longtemps, que la fréquence est plus facile à régler sur un grand que sur un petit réseau.

De plus, la finesse de réglage est - - grossièrement - inversement proportionnelle au degré d'asservissement moyen d'un réseau (ou proportionnelle à la racine carrée de sa rapidité de réponse moyenne $K_{0}$ en réglage accélérométrique). Il s'ensuit qu'on gardera la même finesse de réglage tout en donnant au degré d'asservissement moyen une valeur proportionnelle à la racine carrée de la puissance du réseau (ou en donnant à la rapidité de réponse $K_{n}$ en réglage accélérométrique une valeur proportionnelle à la puissance du réseau).

Pour employer la notion de puissance réglante temporaire (voir note p. 192) on peut dire que, pour une finesse de réglage donnée, la puissance réglante temporairc néccssairc est proportionnclle sculcment à la racinc carréc de la puissance totale du réseau. C'est la raison pour laquelle, malgré une proportion relativement considérable de groupes ne participant pas au réglage, la fréquence du réseau français est suffisamment bien réglée, dans les circonstances normales.

Remarque I. - Dans tout ce qui précède, nous avons implicitement supposé que les appareils utilisateurs étaient sans inertie. Or, en fait, certains sont dotés d'inertie. Il est facile de montrer que, pour en tenir compte, il faut ajouter à la moyenne $\tau$ du temps de lancer des groupes moteurs la moyenne $\tau^{\prime}$ des temps de lancer (défnis de la même manièrc) des appareils utilisateurs. Mais il ne faut pas oublier de tenir compte, dans le calcul de $\tau^{\prime}$. des nombreux appareils récepteurs sans inertie, ce qui contribue à diminuer considérablement la valeur de $\tau^{\prime}$. Encore qu'on ne pussède guère de statistique à ce sujet, il ne scmble pas que cela conduisc à majorcr frelivement de plus de 10 à $20 \%$ le temps de lancer $\tau$ des formules de stabilité. Cela contribue seulement à donner un certain coefficient de sécurité à ces formules.

Remarque II. - Les caracléristiques puissance-fréquence du réseau récepteur et de la lurbine sont telles qu'il y a un phénomène d'autostabilisation du système el que, par suite, les conditions de stabilité sont moins sévères que 
celles que nous avons trouvées. Mais le calcul montre que, là encore, le gain est petit. Cet effet s'ajoute donc au précédent pour donner un coefficient de sécurité aux formules établies.

CAS OU LES HYPOTHESES FAITES NE SONT PAS RÉALISEEES. - Les hypothèses relatives à la non-élasticité de l'eau et des conduites, et à la rigidité du couplage électrique entre groupes moteurs et utilisateurs, ne sont pas toujours réalisées.

En ce qui concerne la mon-élasticité de l'eau et des conduites, cette hypothèse n'exclut que les très hautes chutes et M. GAdEN a mis sur pied une théorie valable aussi dans ce cas, et qui aboutit aux mêmes conclusions.

En ce qui concerne l'hypothèse de la rigidité du couplage électrique, il est difficile à un hydraulicien de se prononcer sans le secours d'électriciens spécialisés. Essayons, cependant, de préciser cette notion de rigidité.

En toute rigueur, dire qu'une interconnexion est rigide, c'est dire que l'angle de phase entre le générateur et le récepteur est constant. En fait, lors d'un changement de régime, l'angle de phase ne passe pas instantanément d'une valeur à une autre, et subit des oscillations. Si ces oscillations sont rapides vis-à-vis des oscillations propres des groupes munis de leurs régulateurs (plusieurs secondes) on pourra encore dire que tout se passe comme si l'interconnexion était rigide; mais si ces oscillations ont des périodes de même ordre que celles des groupes, l'hypothèse de la rigidité n'est plus valable. Or, la période d'oscillation de l'angle de phase est d'autant plus petite que l'angle de phase est plus éloigné de $\frac{\pi}{2}$ (limite de stabilité statique). Donc l'hypothèse de la rigidité de l'interconnexion sera d'autant mieux réalisée que les lignes seront plus éloignées de leur limite de stabilité statique, donc moins chargées.

Considérons un réseau d'interconnexions rigides sur lequel est couplé d'une manière plus lâche un groupe hydroélectrique. Il semble évident que si ce groupe est instable en réseau séparé, il y aura risque de décrochage sur la ligne d'interconnexion qui le relie au réseau général stable. Même s'il n'y a pas de décrochage, il participera mal au réglage de la fréquence du réseau général.

Un grand réseau peut être considéré comme constitué par des réseaux partiels à l'intérieur desquels le couplage peut être considéré comme rigide, reliés entre eux par des lignes d'interconnexions non rigides. Il est donc logique de penser qu'il y aurait intérêt à ce que chaque réseau partiel soit capable d'assurer la stabilité de la puissance qu'il fournit, ainsi qu'un bon réglage de sa fréquence, puisqu'il ne peut compter, que dans une mesure que nous ne connaissons pas, sur l'éventuelle marge de stabilité des réseaux voisins. Il y a là une question qui aurait besoin d'être élucidée par un calcul fait en collaboration par des hydrauliciens et des électriciens.

\section{CONCLUSIONS GÉNÉRALES}

Nous avons, au cours de cette étude, donné les conditions de stabilité en réseau séparé de groupes hydroélectriques contrôlés par des régulateurs de divers types. Nous avons montré les analogies et les différences de leurs actions.

Nous avons montré l'influence que le comportement stable ou instable d'un groupe en réseau séparé a, après couplage, sur le comportement du réseau général.

D'où l'intérêt qu'il y aurait à établir une statistique de caractéristiques de régulation des groupes (puissance, temps de lancer, temps caractéristiques des conduites, rapidité de réponse, degrés d'asservissement) et, notamment, une statistique des groupes sur limiteur d'ouverture; il serait, en particulier, utile de connaître la puissance totale de ces groupes, comparée à la puissance totale du réseau. Ceci nous donnerait une idée de la marge de stabilité dont dispose le réseau français.

L'équipement hydroélectrique de notre pays, encore que les possibilités d'équipement de hautes chưtes nouvelles ne soient pas encore épuisées, va s'engager tout naturellement dans la voie de l'exploitation des basses chutes, sous forme de groupes Kaplan de grande puissance. Pour ces groupes, la valeur de $\Theta$ peut atteindre et dépasser la valeur 3, par suite de leur faible hauteur de chute, alors que les valeurs habituellés de $\Theta$ sont de l'ordre de 1 à 1,5 . Les temps de lancer des groupes sont de l'ordre de 5 à $10^{\prime \prime}$ et n'augmentent pas en proportion de leur puissance (pour des raisons de construction des alternateurs et parce qu'il n'est guère possible d'adjoindre un volant à des groupes à axes verticaux).

Dès lors, les condilions de stabilité montrent que pour maintenir la stabilité de tels groupes, lorsquilis travailleronl en réeeau séparć, il faudra donner à leurs degrés d'asservissement (en réglage tachymótriquc) dcs valcurs beaucoup plus grandes, et à leurs rapidilés de réponses (en réglage accélérnmétrigue) des valeurs beaucoup plus faibles 
que celles auxquelles nous sommes habitués actuellement. Nous avons vu, par exemple, que lorsque la valeur de $\Theta$ est multipliée par 2, cela conduit à multiplier par 2 le degrá d'asservissement ou à diviser par 4 la rapidité de réponse (en réglage accélérométrique). Ceci a pour effet de multiplier approximativement par 2 l'écart de fréquence qui suit une variation donnée de charge, donc cela diminue la finesse de réglage de la fréquence en réseau séparé : c'est dans la nature des choses et nous n'y pouvons rien. Il faut s'y résoudre et ne pas demander un bon réglage de la fréquence en réseau séparé, à des groupes placés dans de mauvaises conditions hydrauliques, en ce qui concerne l'inertie de l'eau ( $\Theta$ grand).

Cependant, le travail de ces groupes en réseau séparé sera exceptionnel et, le plus souvent, ils seront couplés sur le réseau général. Dans ces conditions, deux cas sont à envisager :

a) Une statistique ayant montré que la marge de stabilité du réseau général existant était suffisante, on pourra, par exemple, supprimer ou diminuer, après couplage, l'asservissement de ces groupes, ce qui leur permettra de participer convenablement au réglage de la fréquence du réseau général, tout en diminuant la stabilité de ce dernier. Ceci n'est possible que si le couplage du groupe considéré avec le réseau général peut être considéré comme rigide.

b) Soit que la statistique ait montré que la marge de stabilité du réseau général existant n'était pas suffisante, soit que l'on considère que le nombre de groupes régleurs e t déjà suffisant pour régler convenablement la fréquence du réseau général - on couplera le groupe avec son degré d'asservissement qui contribuera ainsi à la stabilité de l'ensemble - Cela augmentera le degré d'asservissement moyen du réseau, mais nous avons vu qu'on pouvait se le permettre dans une certaine mesure, sans diminuer la finesse de réglage de la fréquence.

Notons que la question ne se pose guère si le groupe est au fil de l'eau, ce qui est fréquemment le cas des basses chutes. Alors, lorsquilil travaillera couplé sur le réseau, on aura intérêt à le placer sur limiteur d'ouverture asservi au niveau amont. Mais il ne faudra pas oublier que si l'on se met à construire de plus en plus des groupes qui, par nature, sont incapables ou peu capables de participer au réglage de la fréquence - soit parce que leur condition d'exploitation (fil de l'eau) ne le leur permet pas, soit que les conditions hydrauliques (inertie de l'eau) les handicapent - on devra corrélativement en construire quelques-uns capables d'y participer, malgré l'influence salutaire - que nous avons analysée - de l'augmentation de la puissance du réseau sur le maintien de la fréquence.

On ne devra admettre pour un groupe une grande valeur de $\Theta$, que lorsqu'il sera impossible de faire autrement, surtout dans les pays où l'équipement hydroélectrique est encore à l'état embryonnaire, car cela hypothéquerait l'avenir. Notamment, il sera prudent de ne pas diminuer outre mesure, sous prétexte d'économie, le diamètre des conduites, et ne pas supprimer inconsidérément une cheminée d'équilibre, même si les conditions de surpression ou de survitesse admises le permettent.

Lorsqu'une installation comporte une cheminée d'équilibre convenablement dimensionnée, le temps caractéristique $\Theta$ s'entend pour toute la portion de conduite à l'aval de la cheminée. La surpression de la cheminée introduit le $\Sigma$ LV de toute la portion amont, ce qui augmente la valeur de $\Theta$; il y a, d'autre part, lieu de remarquer que la solution qui consiste à employer un déchargeur, si elle diminue la surpression maximum à craindre pour une fermeture de la turbine en un temps donné, ne diminue nullement la valeur du $\Theta$ d'une installation : lors des petits mouvements du réglage, le déchargeur ne fonctionne pas; s'il fonctionnait, cela entraînerait une perte continue d'eau, donc une diminution inacceptable du rendement de l'installation. Il y a là une infériorité du déchargeur sur la cheminée d'équilibre, en ce qui concerne la stabilité du réglage.

Nous pensons avoir attiré, par ces trois articles, l'attention des techniciens sur un aspect peu connu de la régulation des groupes hydroélectriques dû à l'inertie du fluide moteur employé. Cette inertie les rend moins aptes que la plupart des autres générateurs, à régler la fréquence d'un réseau. Ceci est d'ailleurs dû à la nature même des choses et ne dépend pas du système de réglage employé. Nous attirons l'attention des lecteurs sur le fait que ce n'est pas la valeur de la surpression maximum atteinte lors d'une fermeture du vannage à sa vitesse maximum qui caractérise l'effet de l'inertie de l'eau sur la stabilité, mais seulement le temps $\Theta=\frac{\Sigma L V}{g . H}$. Nous espérons que nous aurons aiguisé la curiosité des chercheurs et notamment des électriciens qui, connaissant mieux maintenant dans quelles ronditions s'effectue la régulation des groupes hydroélectriques, pourronl éludier en connaissance de cause les réartions du fonctionn s hydranliciens leurs desideratia à 